\title{
Psychophysiological responses to visceral and somatic pain in functional chest pain identify clinically relevant pain clusters
}

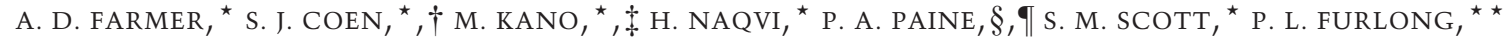 \\ S. L. LIGHTMAN, $\dagger \dagger$ C. H. KNOWLES ${ }^{\star} \&$ Q. AZIZ* \\ ${ }^{\star}$ Centre for Digestive Diseases, Blizard Institute, Wingate Institute of Neurogastroenterology, Barts and the London School \\ of Medicine \& Dentistry, Queen Mary University of London, London, UK \\ $\dagger$ Department of Neuroimaging, Institute of Psychiatry, King's College London, London, UK \\ $\ddagger$ Behavioural Medicine, Tohoku University Graduate School of Medicine, Sendai, Japan \\ $\S$ Gastrointestinal Sciences, University of Manchester, Manchester, UK \\ qSalford Royal Foundation NHS Trust, Salford, UK \\ **Aston Brain Centre, School of Life and Health Sciences, Aston University, Birmingham, UK \\ $\dagger$ †Henry Wellcome Laboratories for Integrative Neuroscience \& Endocrinology, University of Bristol, Bristol, UK
}

\section{Key Messages}

- Functional chest pain of presumed esophageal origin is a complex but incompletely understood disorder.

- Here we demonstrate differences in personality and anxiety traits and pain tolerance thresholds to visceral and somatic pain between patients and healthy controls.

- Patients also largely segregate into a specific "pain cluster."

\begin{abstract}
Background Despite chronic pain being a feature of functional chest pain (FCP) its experience is variable. The factors responsible for this variability remain unresolved. We aimed to address these knowledge gaps, hypothesizing that the psychophysiological profiles of FCP patients will be distinct from healthy subjects. Methods 20 Rome III defined FCP patients (nine males, mean age 38.7 years, range 28-59 years) and 20 healthy age-, sex-, and ethnicity-matched controls (nine males, mean 38.2 years, range 24-49) had anxiety, depression, and personality traits measured. Subjects had sympathetic and parasympathetic nervous system parameters measured at
\end{abstract}

Address for Correspondence

Prof Qasim Aziz, PhD, MRCP, Wingate Institute of Neurogastroenterology, 26 Ashfield Street, London E1 2AJ, UK.

Tel: +44 (0) 2078822648; fax: +44 (0) 2078825640;

e-mail: a.farmer@qmul.ac.uk

Received: 27 July 2013

Accepted for publication: 15 September 2013 baseline and continuously thereafter. Subjects received standardized somatic (nail bed pressure) and visceral (esophageal balloon distension) stimuli to pain tolerance. Venous blood was sampled for cortisol at baseline, post somatic pain and post visceral pain. Key Results Patients had higher neuroticism, state and trait anxiety, and depression scores but lower extroversion scores vs controls (all $p<0.005)$. Patients tolerated less somatic $(p<0.0001)$ and visceral stimulus ( $p=0.009)$ and had a higher cortisol at baseline, and following pain (all $p<0.001)$. At baseline, patients had a higher sympathetic tone ( $p=0.04)$, whereas in response to pain they increased their parasympathetic tone $(p \leq 0.008)$. The amalgamating the data, we identified two psychophysiologically distinct 'pain clusters'. Patients were overrepresented in the cluster characterized by high neuroticism, trait anxiety, baseline cortisol, pain hypersensitivity, and parasympathetic response to pain (all $p<0.03$ ). Conclusions \&) Inferences In future, such delineations in FCP populations may facilitate individualization of treatment based on psychophysiological profiling. 
Keywords Functional chest pain of presumed esophageal origin, pain clusters, pathophysiology.

\section{INTRODUCTION}

Functional chest pain (FCP) of presumed esophageal origin is characterized by recurrent unexplained midline chest pain. The Rome III diagnostic criteria include at least 3 months of symptoms, with onset at least 6 months prior to diagnosis, in the absence of another cause. ${ }^{1}$ Patients often demonstrate a significant reduction in quality of life, have recourse to disproportionately high healthcare utilization, which itself is often manifested in recurrent negative investigations across a multitude of medical specialties, and are frequently recalcitrant to standard therapies. ${ }^{2}$

The pathophysiological mechanisms proposed to account for the genesis, and maintenance, of symptoms in FCP are incompletely understood. ${ }^{3}$ To date, three mechanisms in particular have been subject to objective evaluation. Firstly, esophageal hypersensitivity, first described in 1986 by Richter et al. and subsequently confirmed by others, is considered to be a pathophysiological feature albeit with insufficient specificity and sensitivity for routine diagnostic use in clinical practice. ${ }^{4-6}$ Nevertheless, this observation has spawned further research suggesting that a combination of an increase in afferent pathway sensitivity, abnormal cortical processing or pain hypervigilance may account for this epiphenomenon. ${ }^{7}$ Secondly, the stress-responsive physiological systems, namely, the autonomic nervous system (ANS), comprising of the parasympathetic (PNS) and sympathetic nervous systems (SNS), and the hypothalamic-pituitary-adrenal (HPA) axis, act as brain-body interfaces and are thus critical components of physiological adaption in response to changes in the external and internal environments. It is therefore not surprising that dysfunction within these systems has been implicated as a pathophysiological feature in a number of functional disorders including NCCP. ${ }^{8}$ Finally, up to $61 \%$ of FCP patients display a degree of psychological comorbidity, which itself may enhance esophageal perception possibly through hypervigilance. ${ }^{9,10}$ Whether psychiatric comorbidity is a primary cause, a predisposing factor, a comorbid illness or indeed a sequelae, remains to be fully determined.

Therefore, research is warranted to further characterize its many pathophysiological features within a single paradigm. Arguably, almost on a de rigueur basis, the majority of the previous studies have largely examined these features in isolation of one another and thus such a reductionist approach has constrained our understanding of their corelationships within possible 'pain clusters'. In a recent study, we have demonstrated, in health, the existence of two temporally stable multifaceted pain clusters. ${ }^{11}$ The first of these, accounting for $\sim 1 / 3$ rd of the healthy population, at baseline had higher neuroticism/anxiety scores, SNS tone and cortisol levels but during pain had lower pain thresholds with a concomitant increase in PNS tone. The second cluster, accounting for the remainder, had the converse profile at baseline and during pain. Furthermore, these clusters exhibited differences in polymorphisms of the serotonin transporter linked polymorphic region and brain processing. Thus, the primary aim of our study was to identify key psychophysiological profiles in patients with FCP within a single experimental protocol and we reasoned that psychophysiological characteristics of the first pain cluster we have previously described in health would be more prevalent in the patient population.

\section{MATERIALS AND METHODS}

\section{Subjects}

20 FCP patients, defined according to the Rome III criteria, and 20 healthy age-, sex-, ethnicity-matched healthy controls, separate from our previous study, were enrolled into the current study. ${ }^{1}$ Patients were identified from the gastrointestinal (GI) physiology database at the Royal London Hospital and healthy controls were recruited, on an approximate matched aged basis, from the residents of the surrounding geographical area. Within 12 months of the study, all patients had a negative cardiac evaluation (either a negative exercise tolerance test or coronary angiogram) with normal esophageal motility demonstrated on high-resolution manometry, normal $24 \mathrm{~h}$ pH-metry, off antisecretory therapy, and a normal esophago-gastro-duodenoscopy with normal biopsies from the mid- and distal esophagus. Patients were excluded if they fulfilled another Rome III diagnosis for a separate functional GI disorder (FGID). All subjects were naïve to the experimental protocol but received written information beforehand and provided written informed consent. Females were studied in the follicular phase of their menstrual cycle. Subjects were excluded if they were taking any analgesics, centrally acting medications or those influencing autonomic responses. Current smokers (six patients [four males], zero healthy controls) were asked not to smoke for $24 \mathrm{~h}$ before the study. Subjects were asked to refrain from alcohol consumption for $24 \mathrm{~h}$ prior to the study. All subjects were screened for subclinical anxiety and depression using the validated Hospital Anxiety and Depression Scale (HADS) and healthy subjects were excluded if their scores exceeded 7 on either anxiety (HADS-A) or depression (HADS-D) scale. ${ }^{12}$ All subjects were screened for comorbid chronic pain/somatoform disorders. As several measures in the study were questionnaire based, those who exceeded a self-deception score, as assessed by the Weinberger Adjustment Inventory, were excluded from the analysis thus ensuring response integrity. ${ }^{13}$ These studies were approved by the East London and the City Ethics Committee 2 (Ref: 08/H0703/47). 


\section{Personality and anxiety measures}

The validated big five inventory (BFI) was used to measure the personality traits of neuroticism (BFI-N) and extroversion (BFI-E). ${ }^{14}$ State (STAI-S) and trait (STAI-T) anxiety was assessed using the validated Spielberger State-Trait Anxiety Inventory. ${ }^{15}$ These measures were chosen based on our previous study. ${ }^{11}$

\section{Pain induction measures}

Visceral pain induction Subjects were intubated, without local anesthetic, with an esophageal catheter (Sandhill Scientific, Oxford, UK), containing a silicone balloon mounted $1 \mathrm{~cm}$ from the distal catheter tip, which was positioned in the distal esophagus, $34 \mathrm{~cm}$ ab nares. Painful stimulation was achieved by manually inflating the balloon at a rate of $5 \mathrm{mls} / \mathrm{s}$ to the subject's pain tolerance threshold (PTT), which was defined as the point at which the subjects could not tolerate any further increase in stimulus intensity. The catheter was secured in position using adhesive tape (Micropore, 3M Healthcare, Bracknell, UK) applied to the subject's nose, upper lip, and face to minimize any distal displacement of the catheter during pain induction. Somatic pain induction - a strain gauge (Mecmesin, Oxford, UK) was mounted onto a spring-loaded device such that the extension probe incorporated a thin blunt rod (Medical Physics Department, Hope Hospital, Manchester, UK). ${ }^{14}$ This device was applied to the right thumbnail, $5 \mathrm{~mm}$ from the cuticle, producing a stimulus of nail bed force ranging from touch to painful. Force was measured in Newtons (N). Each painful somatic stimulus was achieved by applying nail bed force manually to the subject's PTT.

\section{ANS measures}

Blood pressure Digital arterial blood pressure (BP) was measured non-invasively using the validated photoplethysmographic technique (Portapres, Amsterdam, The Netherlands). ${ }^{16,17}$

Skin conductance responses Skin conductance is a putative sympathetic 'emotional sudomotor' measure response within milliseconds to threatening stimuli. ${ }^{18}$ Skin on the distal digit pulp of the right index and ring fingers was wiped with water and allowed to dry. In each subject, skin conductance electrodes were then attached and the skin conductance level was zeroed using a commercially available bioamp (Powerlab, AdInstruments, Oxford, UK). The mean skin conductance response (SCR) was extracted and analyzed offline.

Heart rate, cardiac vagal tone, and cardiac sensitivity to the baroreflex Electrocardiogram (ECG) electrodes (Ambu Blue Sensor P, Ballerup, Denmark) were placed in right and left subclavicular areas and cardiac apex. The ECG was acquired using a commercially available biosignals acquisition system (Neuroscope ${ }^{\mathrm{TM}}$, Medifit Instruments, Enfield, UK). The Neuroscope measures brainstem PNS efferent activity, known as cardiac vagal tone $(\mathrm{CVT})$, in real time and is measured on a validated linear vagal scale (LVS), where 0 represents full atropinization. ${ }^{19}$ The Neuroscope also incorporates beat-to-beat R-R interval and mean BP (MBP) into an algorithm on a 10-s cycle, calculating cardiac sensitivity to the baroreflex (CSB), an indirect measure of PNS afferent activity. ${ }^{20}$ These measures are described in detail elsewhere, ${ }^{14}$ but in contrast to power spectral analysis of heart rate variability, are validated for time epochs of less than 1 min. ${ }^{14}$
Pain evoked dynamic peri-stimulus pain-related change in autonomic variables was examined by calculating $\Delta$ change by comparing the $30 \mathrm{~s}$ pre- $v s$ the $30 \mathrm{~s}$ poststimulus; time points considered to demonstrate the maximal change in these parameters and thus utilizing the improvements in temporal resolution conferred by the Neuroscope. ${ }^{14}$ Autonomic parameters were recorded according to internationally agreed guidelines. ${ }^{21}$

\section{Serum cortisol measurement}

Serum total cortisol was assayed by the Blood Services Department at the Royal London Hospital, London, UK, using a chemiluminescence immunoassay (Nichols Advantage, San Juan, CA, USA). In this study, $90-95 \%$ of total cortisol in the plasma is inactive and bound to cortisol binding globulin and to a lesser extent albumin. The remaining $5 \%$ is active and available for access to extracellular fluid and intracellular glucocorticoid receptors and is a validated measure of HPA axis activation. ${ }^{22}$

\section{Experimental protocol}

All subjects were studied in the afternoon (from 14:00 to 16:00 h) in a temperature-controlled $\left(20-22{ }^{\circ} \mathrm{C}\right)$, quiet laboratory. Subjects completed the questionnaires and were reclined at $45^{\circ}$ on a couch. Subsequent to the attachment of ANS recording equipment, $5 \mathrm{~min}$ of baseline autonomic data (resting/no stimulation) was acquired. Venous blood was sampled for baseline serum cortisol. Seven somatic stimuli were administered to PTT, with an interstimulus interval (ISI) of $2 \mathrm{~min}$, followed by a 15 -min rest period. Subjects were then intubated with the esophageal catheter and allowed to rest for $15 \mathrm{~min}$ and subsequently received seven esophageal stimuli, with an ISI of $2 \mathrm{~min}$, to PTT. Subjects rated each stimulus for intensity ( 0 no sensation to 10 worst imaginable intensity) and unpleasantness $(0$ no unpleasantness to 10 worst imaginable unpleasantness) on a verbal report scale. Somatic and visceral stimulus toleration and ratings were derived from the mean for each. Subjects received a verbal cue $5 \mathrm{~s}$ prior to each stimulus delivery to attempt to standardize any effects of anticipation. Venous blood was further sampled for cortisol $2 \mathrm{~min}$ after the final somatic and visceral stimulus. The experimental protocol is summarized in Fig. 1.

\section{Data analysis}

Categorical variables are expressed as percentages and Chi-squared tests/Fisher's exact tests were used to compare groups. Continuous variables were expressed as mean \pm SEM. Autonomic nervous system and HPA axis data were normally distributed and group comparison was undertaken using a twotailed Student's $t$-test. Mann-Whitney $U$-tests were used to compare personality trait scores. To potentially stratify pain clusters, two-step cluster analysis, amalgamating both the patient and healthy control data, was used. Two-step cluster analysis is a validated robust multivariate statistical method that can analyze large datasets. ${ }^{23}$ In the first step, cases are assigned into preclusters and these preclusters are treated as single cases in the second step where a hierarchical algorithm is used to cluster the preclusters. The two-step cluster does not require the investigator to make any assumptions regarding the optimum number of clusters, as it generates these automatically. ${ }^{24}$ The cluster quality is evaluated by the silhouette coefficient, a measure of cohesion and separation, with a coefficient $>0.5$ representing good separation. ${ }^{25}$ Input factors of BFI-N, BFI-E, baseline CVT, and change in CVT to visceral and somatic pain were defined $a$ priori based on 

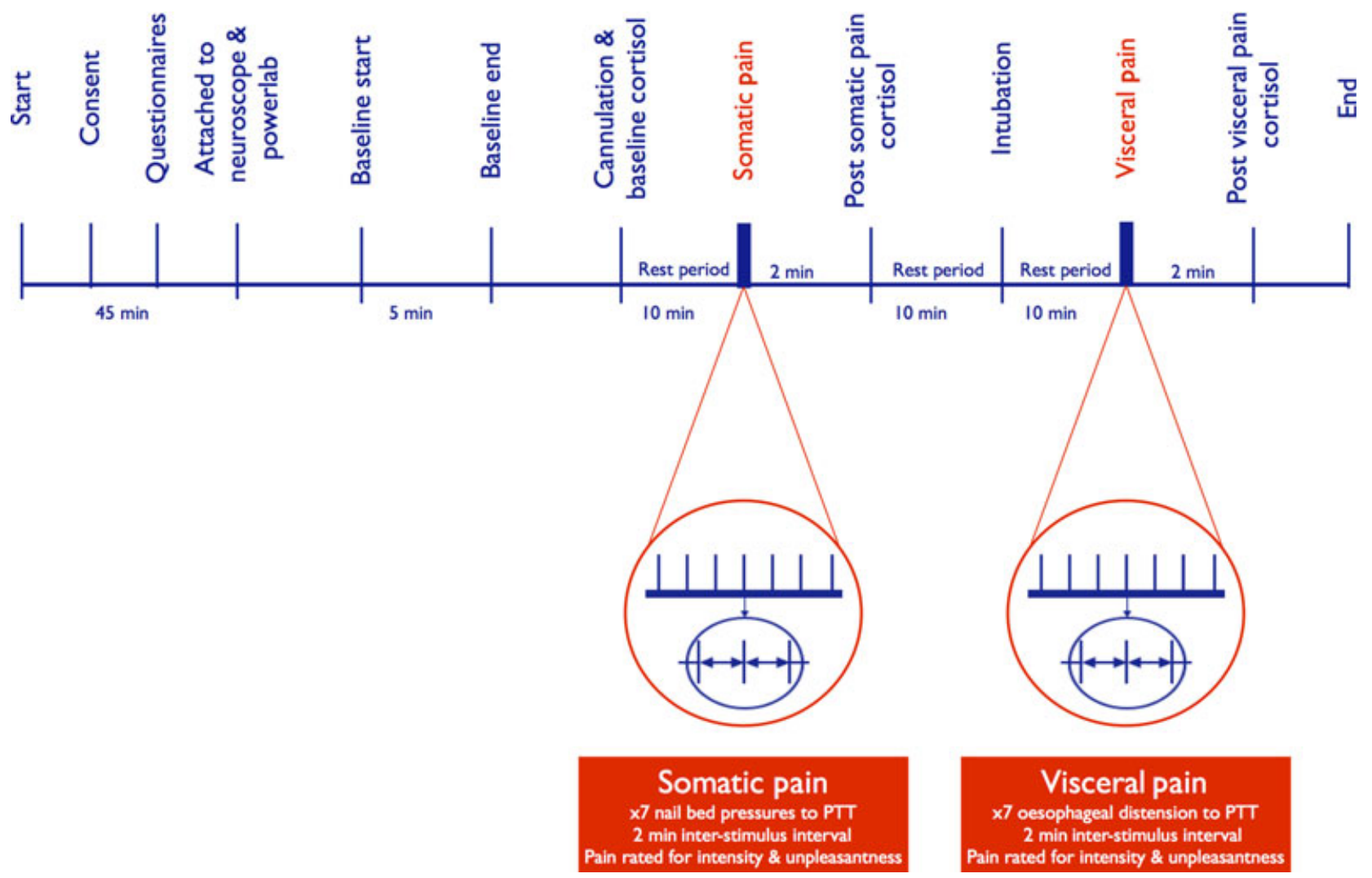

Figure 1 A schematic summary of the psychophysiological experimental protocol.

our previous studies. ${ }^{11,26}$ There is no consensus as to the optimum sample size to perform a cluster analysis ${ }^{27}$ but it is recommended that minimal sample size includes no less than $2^{\mathrm{k}}$ cases $(\mathrm{k}=$ number of input variables), in which this study exceeded. ${ }^{28}$ All tests were two tailed, and $p<0.05$ was adopted as the statistical criterion. Analyses were performed using proprietary software (GraphPad Prism 5, La Jolla, CA, USA and SPSS 18, IBM, New York, NY, USA).

\section{RESULTS}

Twenty patients (nine males, mean age of 38.7 years, range 28-59 years) and 20 healthy volunteers were recruited to the study (nine males, mean age of 38.2 years, range 24-49 years). All subjects were caucasian. Two patients did not complete the study. One (male, aged 28.4 years) was excluded as he exceeded the self-deception score, and the other (female, aged 44.2 years) did not tolerate esophageal intubation. All healthy volunteers completed the study.

\section{Psychological characteristics}

In comparison with healthy controls, patients had higher anxiety and depression scores, as assessed by the HADS, higher neuroticism, state and trait anxiety with
Table 1 The psychological state and trait characteristics of patients in comparison with healthy controls

\begin{tabular}{llrl}
\hline & $\begin{array}{l}\text { Patients } \\
(n=18) \\
\text { Mean } \pm \text { SEM }\end{array}$ & $\begin{array}{l}\text { Controls } \\
(n=20) \\
\text { Mean } \pm \text { SEM }\end{array}$ & $p$ value \\
\hline HADS - A & $4.65 \pm 0.47$ & $2.45 \pm 0.34$ & 0.0005 \\
HADS - D & $4.82 \pm 0.63$ & $1.9 \pm 0.36$ & 0.0002 \\
BFI-N & $3.67 \pm 0.25$ & $2 \pm 0.36$ & 0.0001 \\
BFI-E & $3.04 \pm 0.42$ & $4.2 \pm 0.23$ & 0.003 \\
STAI-T & $43.5 \pm 2.47$ & $28.4 \pm 8.5$ & 0.009 \\
STAI-S & $41.7 \pm 2.6$ & $27.7 \pm 2.2$ & 0.0007 \\
\hline
\end{tabular}

lower extroversion scores, see Table 1. The psychological characteristics of the patients who completed the study were not significantly different to those that were either excluded/did not tolerate esophageal intubation.

\section{Behavioral responses to pain}

The mean somatic stimulus tolerated by patients was $48.4 \mathrm{~N} \pm 3.4$ vs $70.61 \mathrm{~N} \pm 3.6$ by the controls $(p<0.0001)$. The mean visceral stimulus tolerated by patients was $30 \mathrm{mls} \pm 1.8$ vs $50.9 \mathrm{mls} \pm 6.9$ for the controls $(p=0.009)$. There was no difference in the 
somatic pain intensity and unpleasantness ratings between patients and controls $17.72 \pm 0.29$ VS $7.62 \pm 0.31, p=0.81$ and $6.26 \pm 0.24$ vs $6.28 \pm 0.3$, $p=0.94$, respectively). Similarly, there was no difference in the visceral pain intensity and unpleasantness rating between patients and controls (7.26 \pm 0.25 vs $7.62 \pm 0.47$, $p=0.51$ and $7.44 \pm 0.28$ vs $7.39 \pm 0.44, p=0.93)$.

\section{Hypothalamic-pituitary-adrenal axis responses to pain}

Serum cortisol was higher in patients at baseline and following somatic and visceral pain, see Fig. 2.

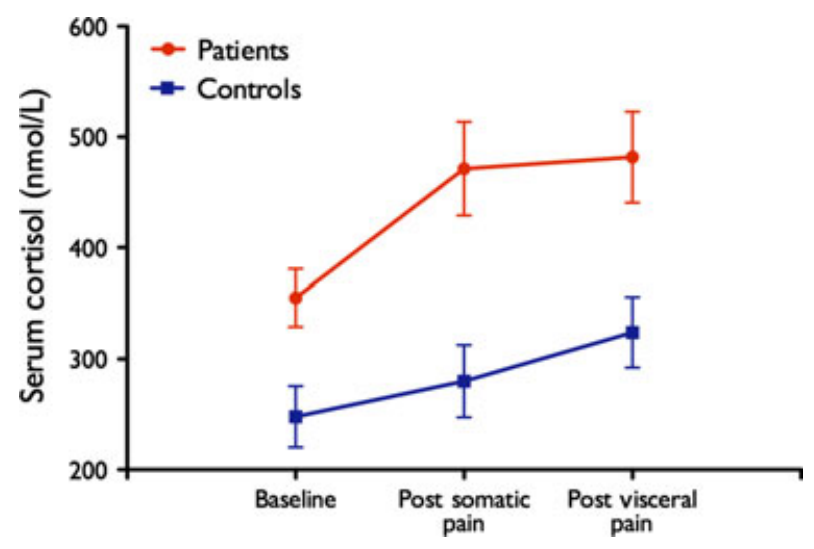

Figure 2 Serum cortisol at baseline, post somatic pain and post visceral pain in patients and controls, all differences $p<0.01$.
Although there was no significant difference in the absolute increase in cortisol post pain between patients and controls, the levels in the patients began at very high values for the afternoon, and unlike the levels in control subjects reached concentrations associated with a significant stress response. ${ }^{29}$

\section{Baseline ANS parameters}

At baseline, patients had an elevated heart rate $(75.9 \pm 4.7$ VS $67.2 \pm 1.7, p=0.04)$ and SCR $(4.2 \pm 0.9$ vs $2.05 \pm 0.22, p=0.04)$ with lower CVT $(5.5 \pm 0.84$ VS $11.76 \pm 1.6, p=0.003)$ and CSB $(4.79 \pm 0.92$ vs $8.76 \pm 1.27, p=0.02)$ in comparison with controls. At baseline, no differences were apparent in BP.

\section{ANS responses to pain}

In response to somatic pain, patients' heart rate increased less than controls, with a concomitant reduction in systolic $\mathrm{BP}$ and a marked increase in parasympathetic tone (CVT and CSB), which was not evident in the controls (see panels $\mathrm{A}$ and $\mathrm{B}$, respectively, see Fig. 3). To visceral pain, patients compared to controls slowed their heart rate, decreased systolic $\mathrm{BP}$, increased PNS tone (CVT and CSB) (see panels C and $\mathrm{D}$, respectively, see Fig. 2), but withdrew SNS tone (SCR), see Table 2.
Figure 3 Peri-stimulus dynamic changes in efferent and afferent parasympathetic tone (CVT and CSB, respectively) to somatic pain (A and B) and to visceral pain (C and D) between patients and controls. Patients increased their parasympathetic tone to somatic and visceral pain whereas controls withdrew it. * Statistically significant at $p<0.001$.
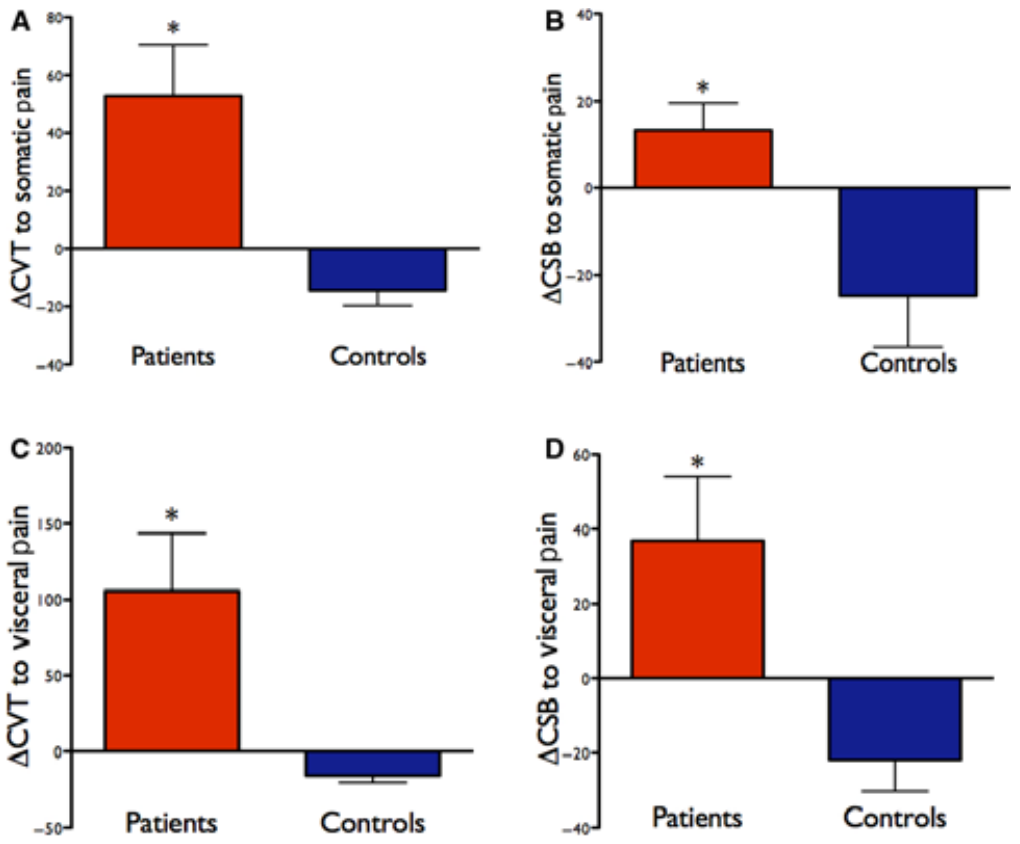
Table 2 Dynamic peri-stimulus change in autonomic parameters to somatic and visceral pain in patients and controls

\begin{tabular}{|c|c|c|c|}
\hline & $\begin{array}{l}\text { Patients } \\
\text { Mean }( \pm \text { SEM) }\end{array}$ & $\begin{array}{l}\text { Controls } \\
\text { Mean }( \pm \text { SEM) }\end{array}$ & $p$ value \\
\hline \multicolumn{4}{|c|}{ Peri-stimulus dynamic changes in ANS parameters to somatic pain } \\
\hline$\Delta \mathrm{HR}(\% \Delta \mathrm{bpm})$ & $0.27 \pm 1.4$ & $8.3 \pm 2.6$ & $0.01^{\star}$ \\
\hline$\Delta \mathrm{SBP}(\% \Delta \mathrm{mmHg})$ & $-0.27 \pm 0.6$ & $2.9 \pm 1.1$ & $0.03^{\star}$ \\
\hline $\begin{array}{l}\Delta \text { Diastolic BP } \\
(\% \Delta \mathrm{mmHg})\end{array}$ & $1.07 \pm 0.68$ & $2.8 \pm 1.08$ & 0.18 \\
\hline$\Delta \mathrm{MBP}(\% \Delta \mathrm{mmHg})$ & $1.22 \pm 0.45$ & $3.12 \pm 0.99$ & 0.07 \\
\hline$\Delta \mathrm{CVT}(\% \Delta \mathrm{LVS})$ & $53 \pm 18.6$ & $-14.1 \pm 5.2$ & $0.002^{\star}$ \\
\hline $\begin{array}{l}\Delta \mathrm{CSB}(\% \Delta \Delta \mathrm{RR} / \\
\Delta \mathrm{mmHg})\end{array}$ & $13.24 \pm 6.3$ & $-24.8 \pm 11.7$ & $0.01^{\star}$ \\
\hline$\Delta \mathrm{SCR}(\% \Delta \mu \mathrm{S})$ & $0.09 \pm 1.7$ & $15.88 \pm 1.4$ & $0.007^{\star}$ \\
\hline \multicolumn{4}{|c|}{ Peri-stimulus dynamic changes in ANS parameters to visceral pain } \\
\hline$\Delta \mathrm{HR}(\% \Delta \mathrm{bpm})$ & $-1.69 \pm 1.2$ & $5 \pm 1.35$ & $0.002^{\star}$ \\
\hline$\Delta \mathrm{SBP}(\% \Delta \mathrm{mmHg})$ & $-1.66 \pm 0.9$ & $3.5 \pm 1.4$ & $0.008^{\star}$ \\
\hline $\begin{array}{c}\Delta \text { Diastolic BP } \\
(\% \Delta \mathrm{mmHg})\end{array}$ & $-0.98 \pm 1.1$ & $3.1 \pm 1.66$ & 0.06 \\
\hline$\Delta \mathrm{MBP}(\% \Delta \mathrm{mmHg})$ & $-0.85 \pm 0.9$ & $3.6 \pm 1.7$ & $0.02^{\star}$ \\
\hline$\Delta \mathrm{CVT}(\% \Delta \mathrm{LVS})$ & $105.7 \pm 38$ & $-15.9 \pm 4.5$ & $0.008^{\star}$ \\
\hline $\begin{array}{l}\Delta \mathrm{CSB}(\% \Delta \Delta \mathrm{RR} / \\
\Delta \mathrm{mmHg})\end{array}$ & $36.97 \pm 17$ & $-21.8 \pm 8.5$ & $0.008^{\star}$ \\
\hline$\Delta \mathrm{SCR}(\% \Delta \mu \mathrm{S})$ & $-0.73 \pm 0.2$ & $12.78 \pm 0.8$ & $0.006^{*}$ \\
\hline
\end{tabular}

${ }^{\star}$ Statistically significant.

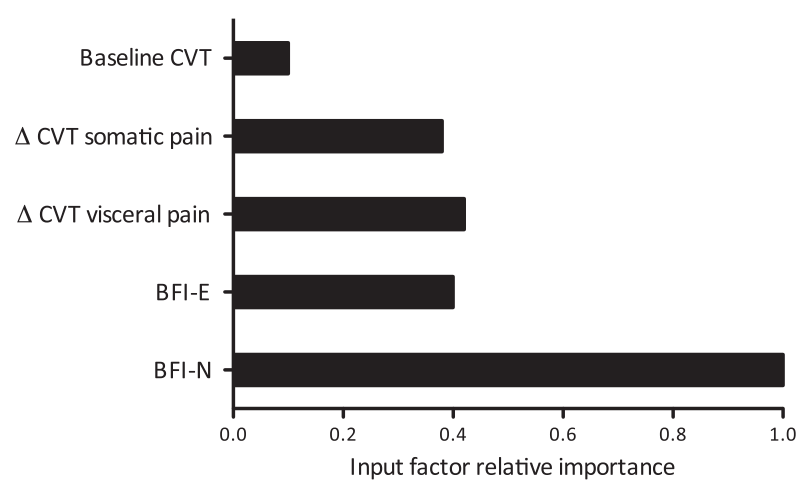

Figure 4 A graphical summary of the relative importance of the input factors (BFI neuroticism, BFI extroversion, change in cardiac vagal tone to somatic and visceral pain and baseline cardiac vagal tone) to the composition of the clusters. For example, BFI neuroticism relative importance was 1.0 compared to baseline cardiac vagal tone of 0.1 suggesting that this psychological trait is $\times 10$ more important in delineating the clusters.

\section{Cluster analysis reveals clinically relevant pain clusters}

The optimum solution for the amalgamated dataset was two clusters, with cluster 1 containing 22 subjects $(57.9 \%)$ and cluster 2 containing 16 subjects $(42.1 \%)$, hereinafter termed pain cluster 1 and pain cluster 2 . The relative predictor importance of the input variables to the two-step cluster analysis is shown in Fig. 4. The cluster silhouette coefficient was 0.61 . The silhouette coefficient maybe interpreted as between -1 , indicating a very poor model, and 1 , indicating an
Table 3 Psychophysiological differences between the pain clusters. Demographic, personality traits, baseline autonomic, visceral, and somatic pain differences between pain clusters 1 and 2 are shown

\begin{tabular}{|c|c|c|c|}
\hline Variable & $\begin{array}{l}\text { Cluster } 1 \\
(n=22) \\
\text { Mean }( \pm \text { SEM) }\end{array}$ & $\begin{array}{l}\text { Cluster } 2 \\
(n=16) \\
\text { Mean }( \pm \text { SEM) }\end{array}$ & $p$ value \\
\hline \multicolumn{4}{|l|}{ Personality traits } \\
\hline BFI neuroticism & $3.7 \pm 0.24$ & $1.95 \pm 0.15$ & $<0.0001^{\star}$ \\
\hline BFI extroversion & $2.92 \pm 0.26$ & $4.36 \pm 0.15$ & $0.0002^{\star}$ \\
\hline STAI-T & $41.6 \pm 32.4$ & $30.3 \pm 2.89$ & $0.007^{\star}$ \\
\hline STAI-S & $39.38 \pm 3.75$ & $31.13 \pm 3.25$ & 0.11 \\
\hline \multicolumn{4}{|l|}{ HPA axis } \\
\hline $\begin{array}{l}\text { Baseline cortisol } \\
\text { (nM) }\end{array}$ & $343.2 \pm 23.25$ & $239.7 \pm 37.32$ & $0.03^{\star}$ \\
\hline $\begin{array}{l}\text { Postsomatic pain } \\
\text { cortisol (nM) }\end{array}$ & $418.1 \pm 22.75$ & $292.6 \pm 54.29$ & $0.04^{\star}$ \\
\hline $\begin{array}{l}\text { Postvisceral pain } \\
\text { cortisol (nM) }\end{array}$ & $449.7 \pm 33.35$ & $316.4 \pm 55.8$ & 0.054 \\
\hline \multicolumn{4}{|l|}{ Baseline ANS } \\
\hline CVT (lvs) & $5.15 \pm 0.68$ & $12.16 \pm 1.5$ & $0.0005^{\star}$ \\
\hline $\operatorname{SCR}(\mu \mathrm{S})$ & $2.08 \pm 0.49$ & $2.78 \pm 0.12$ & 0.43 \\
\hline \multicolumn{4}{|c|}{ Somatic pain ANS responses } \\
\hline Mean stimulus $(N)$ & $57.6 \pm 2.89$ & $72.8 \pm 2.1$ & $0.0001^{\star}$ \\
\hline$\Delta \mathrm{CVT}(\%)$ & $47.67 \pm 14.58$ & $-12.3 \pm 4.6$ & $0.001^{\star}$ \\
\hline$\Delta \mathrm{SCR}(\%)$ & $0.01 \pm 0.9$ & $4.99 \pm 0.9$ & $0.009^{\star}$ \\
\hline \multicolumn{4}{|c|}{ Visceral pain ANS responses } \\
\hline Mean stimulus $(\mathrm{mL})$ & $30.1 \pm 2.11$ & $44.3 \pm 2.45$ & $0.0009^{\star}$ \\
\hline$\Delta \mathrm{CVT}(\%)$ & $94.01 \pm 31.22$ & $-15.43 \pm 3.5$ & $0.002^{\star}$ \\
\hline$\Delta \mathrm{SCR}(\%)$ & $-1.22 \pm 0.42$ & $3.8 \pm 0.9$ & $0.01^{\star}$ \\
\hline
\end{tabular}

*Statistically significant.

excellent model. An average silhouette greater than 0.5 indicates reasonable partitioning of data. ${ }^{30}$ Further comparisons between the two pain clusters are shown in Table 3.

In summary, cluster 1 at baseline had higher neuroticism and trait anxiety scores with lower extroversion scores and also lower PNS tone. In response to somatic pain, cluster 1 tolerated lower stimulus thresholds, had higher cortisol, and displayed a dynamic increase in PNS tone with concomitant SNS withdrawal. Similarly in response to visceral pain, similar patterned responses were observed, although differences in cortisol did not reach statistical significance. Cluster 2 displayed the converse profile. In cluster 1 , there were 15 patients and seven controls and in cluster 2 there were three patients and 13 controls ( $p=0.004$, relative risk $3.6,95 \%$ confidence interval 1.3-10.5). Derived from this data, the probability distribution was highly significant $(p<0.0001)$ with a sensitivity of 0.83 (95\% confidence interval $0.7-0.9)$ and specificity of $0.65 \quad(95 \%$ confidence interval $0.55-0.7)$.

\section{DISCUSSION}

We have demonstrated differences in personality traits, somatic and visceral sensitivity, HPA axis, and ANS 
parameters at baseline and in responses to pain in Rome III defined FCP within a single experiment. Patients had higher anxiety, neuroticism scores, and tolerated less mean somatic and visceral stimulus in comparison with the controls. Furthermore, cluster analysis defined two homogeneous pain clusters, which we believe may have clinical salience. Our discussion will focus on three important aspects to be derived from this study. Firstly, we will examine the pathophysiology of FCP including the role of hyperalgesia, personality traits and HPA axis/ANS responses. Secondly, we shall conjecture as to the importance of the pain clusters with respect to the current literature. Finally, we shall suggest some practical and clinical applications for the future.

We have demonstrated that patients with FCP have reduced tolerance to both somatic and visceral pain thus suggesting a degree of hyperalgesia across these modalities. It is interesting to observe that rating of pain intensity and unpleasantness were not different between patients and healthy controls and is likely to be the consequence of the instruction given to participants to tolerate the stimulus to PTT. However, we would acknowledge that the hyperalgesia observed could be, in part, explained by elevated state anxiety that was observed in the patient group. Our observation of esophageal hyperalgesia is consistent with that of Rao et al. who demonstrated that patients with FCP demonstrate lower esophageal pain thresholds to balloon distension compared to controls. ${ }^{31}$ To date, however, there is a paucity of studies evaluating whether hyperalgesia is limited to the esophagus in FCP per se or is more generalized. In contrast, in patients with irritable bowel syndrome, where visceral hypersensitivity is a frequently reported phenomenon, somatic pain and hyperalgesia often coexist, which may be explained by the presence of concomitant somatic pain disorders such as fibromyalgia. ${ }^{32}$ We have previously shown that $N$-methyl- $D$-aspartate receptor blockade reverses acid induced esophageal and somatic hyperalgesia suggesting that central sensitization is a plausible mechanism for viscerosomatic hyperalgesia. ${ }^{33}$ It is therefore intriguing to speculate that the viscerosomatic hyperalgesia seen in our FCP patients may have a similar mechanistic basis although the influence of psychological factors cannot be discounted.

Although previous work has demonstrated higher levels of anxiety, depression, and affective stress in FCP patients, we have additionally observed higher neuroticism and lower extroversion scores. ${ }^{34}$ Specific personality traits and psychopathology, such as anxiety and somatoform disorders, are overrepresented in patients with FCP. ${ }^{35}$ However, hitherto none of these personality or anxiety traits have been reliably segregated with any objective physiological characteristics in FCP. While neuroticism, extroversion, and anxiety are all traits that are distributed on a continuous scale, and thus do not represent psychopathology per se, it is interesting to note the significant differences do exist between patients and controls.

Dysfunction of the HPA axis is among the most investigated biological risk marker in functional somatic (FSD) and FGID. Although in our current study there was no significant difference in the absolute change in cortisol between the two groups, the patient group started at remarkably high levels of cortisol for the time of day (mid-afternoon) and following the painful stimuli reached levels of cortisol normally only found at the peak of morning pulses or following an acute stressor. ${ }^{36}$ These high levels suggest increased basal activity of the HPA axis with further responsiveness to painful stimuli. It is possible that some of this may reflect the differential state anxiety scores between patients and controls. For example, Ritsner et al. demonstrated in both healthy volunteers and patients with psychiatric comorbidity that baseline cortisol is related to anxiety levels. ${ }^{37}$ In conjunction with other data presented herein, we postulate that a common central pathway may link anxiety levels with activation of the HPA axis. However, these observations must be tempered by a recent metaanalysis which did not find evidence of HPA axis dysfunction across the most common FSD and FGID, although FCP was not included. ${ }^{38}$

We have shown that at baseline patients had higher HR and SCR with lower CVT in comparison with controls, suggesting higher SNS and lower PNS tone. These findings are in agreement with a previous study by Tougas et al., who observed that patients with NCCP, who developed pain following esophageal acidification, demonstrate higher baseline HR and lower vagal tone ${ }^{8}$ but during acid infusion these patients increase their vagal tone in a manner similar to the dynamic ANS responses that were seen in our FCP patients to visceral and somatic painful stimuli. It is plausible to speculate that this transient increase in parasympathetic tone to pain in patients with NCCP/ FCP, who are more sensitive to pain $a b$ initio, could represent a hitherto underrecognized compensatory analgesic mechanism. Indeed, preclinical and clinical evidence is accumulating for such a PNS-mediated analgesic mechanism. For instance, low-energy vagal nerve stimulation, delivered by deep brain or transcutaneous stimulation, produces an analgesic effect. ${ }^{39,40}$ Thus, augmentation of vagal tone, either physiologically, electrically or pharmacologically, may present a 
novel treatment strategy in patients with symptoms unresponsive to standard interventions. ${ }^{41}$

Using cluster analysis, we further demonstrate the presence of two pain clusters. The first of these, accounting for $35 \%$ of our healthy subjects, but over $83 \%$ of FCP patients, can be characterized as having higher neuroticism and anxiety scores with elevated baseline SNS \& HPA axis tone. These subjects had lower pain thresholds and had an attenuated HR response and increased their PNS tone to pain. In contrast, the second cluster, accounting for $65 \%$ of our healthy population, yet less than $17 \%$ of FCP patients, display the opposite profile. The external validity for these pain clusters is derived from two strands of evidence. Firstly, in a previous preliminary study of healthy subjects, we identified two pain clusters with similar psychophysiological characteristics. ${ }^{26}$ Secondly, more recently in a larger cohort of 120 healthy subjects, we have replicated these initial findings and demonstrated temporal stability. ${ }^{11}$ Furthermore, the proportions of healthy subjects that segregated to pain clusters 1 and 2 were similar to our previous published work.

It is interesting to cogitate as to the neurobiological basis of these pain clusters. It can be argued that the pain cluster 1 displayed hypervigilant 'tonic-freeze' response to pain, characterized by an increase in PNS tone, whereas pain cluster 2 mounted a more classical 'fight-flight' response, characterized by an increase in SNS tone. Data from studies of animal behavior surmise that the 'tonic-freeze' response may reflect a more anxious or reactive coping style whereas the 'fight-flight' response is a more proactive coping style. $^{42}$ These differences in coping styles may potentially underpin survival strategies imparted by behavioral flexibility. ${ }^{43}$

It is intriguing that many factors identified in our pain clusters have been implicated as features and risk factors for other chronic pain syndromes. ${ }^{4-46}$ Our study therefore provides preliminary evidence that patients with FCP are overrepresented in the first of these pain clusters and further studies are needed to establish the salience of these in a larger group of patients. Likewise, longitudinal studies in healthy populations are also needed to evaluate whether the factors identified in our first pain cluster do represent a conglomerate risk factor for the development of wider FSD or FGID, particularly when an appropriate biopsychosocial challenge occurs.

It may be argued that the psychophysiological profile we have observed in this study is not specific to FCP per se as our cluster analysis in the current and previous study reveals that some healthy subjects also have a similar profile. However, as mentioned above, the traits observed in cluster 1 appear to be present in approximately one-third of healthy subjects but more than $80 \%$ of patients with FCP. It is intriguing to note that about a third of patients who experience somatic or visceral injury/inflammation, such as thoracic surgery or infectious gastroenteritis, go on to develop chronic pain. ${ }^{47,48}$ It is plausible therefore to speculate that the psychophysiological profile in cluster 1 may represent a biomarker of susceptibility to develop chronic pain when the appropriate environmental challenge occurs and thus this profile is unlikely to be specific for any one visceral or somatic pain syndrome. This speculation will of course require confirmation in future studies.

The potential clinical utility of these pain clusters remains to be fully realized but may be far reaching. ${ }^{49}$ Utilizing such an approach may facilitate the development of aggregated biomarkers, an area that has been recently highlighted by a multinational initiative. ${ }^{50}$ Furthermore, pain clusters may allow the stratification of FCP patients toward an individualization of therapeutic interventions. For example, patients in the first pain cluster, who have higher neuroticism and anxiety scores, may preferentially respond to psychological treatments in comparison with those in the second who may have an enhanced response to pharmacological interventions. Moreover, a similar strategy may be beneficial in other FGID considering the substantial overlap between pathophysiological features and the frequent coexistence of multiple FGIDs within a single patient. ${ }^{51}$ In conclusion, this is the first report examining the salience of pain clusters in a chronic visceral pain syndrome. Further studies are now warranted to address the knowledge gaps that remain, particularly directed toward whether the individualization of stratification of treatment between the pain clusters improves outcomes.

\section{ACKNOWLEDGMENTS}

This research/ADF was funded by a Medical Research Council project grant. QA was principal investigator for Medical Research Council grant no. MGAB1A1R.

\section{FUNDING}

This study was funded by the Medical Research Council, Grant Number MGAB1B1R.

\section{CONFLICTS OF INTEREST}

None of the authors have any conflict of interests to declare. 


\section{AUTHOR CONTRIBUTION}

ADF involved in study concept and design, acquisition of data, analysis and interpretation of data, drafting of the manuscript, critical revision of the manuscript for important intellectual content, statistical analysis, obtained funding; $\mathrm{MK}$ and SJC provided study concept and design, acquisition of data, statistical analysis, critical revision of the manuscript for important intellectual content; $\mathrm{HN}$ involved in acquisition of data, critical revision of the manuscript for important intellectual content; PAP and CHK involved in study concept and design, critical revision of the manuscript for important intellectual content; SMS, SLL, and PLF provided technical or material support, study supervision, critical revision of the manuscript for important intellectual content; QA pioneered study concept and design, was principal applicant on Medical Research Council grant, study supervision, critical revision of manuscript for important intellectual content.

\section{REFERENCES}

1 Drossman DA. Rome III: The Functional Gastrointestinal Disorders, 3rd edn. McLean, VA: Degnon Associates, 2006.

2 Achem SR. Noncardiac chest paintreatment approaches. Gastroenterol Clin North Am 2008; 37: 859-78, ix.

3 Ballantyne J, Fishman S, Bonica JJ. Bonica's Management of Pain, 4th edn. In: Fishman SM, Ballantyne JC, Rathmell JP, eds. Philadelphia, PA and London: Lippincott Williams \& Wilkins, 2009.

4 Richter JE, Barish CF, Castell DO. Abnormal sensory perception in patients with esophageal chest pain. Gastroenterology 1986; 91: 845-52.

5 Nasr I, Attaluri A, Hashmi S, Gregersen $\mathrm{H}$, Rao SS. Investigation of esophageal sensation and biomechanical properties in functional chest pain. Neurogastroenterol Motil 2010; 22: 520-6, e116.

6 Rao SS, Gregersen H, Hayek B, Summers RW, Christensen J. Unexplained chest pain: the hypersensitive, hyperreactive, and poorly compliant esophagus. Ann Intern Med 1996; 124: 950-8.

7 Gregersen H, Drewes AM, Frokjaer JB et al. Mechanism-based evaluation and treatment of esophageal disorders. Ann N Y Acad Sci 2011; 1232: 341-8.

8 Tougas G, Spaziani R, Hollerbach S et al. Cardiac autonomic function and oesophageal acid sensitivity in patients with non-cardiac chest pain. Gut 2001; 49: 706-12.

9 Bass C, Wade C. Chest pain with normal coronary arteries: a comparative study of psychiatric and social morbidity. Psychol Med 1984; 14: 51-61.

10 Bradley LA, Richter JE, Pulliam TJ et al. The relationship between stress and symptoms of gastroesophageal reflux: the influence of psychological factors. The American journal of gastroenterology 1993; 88: 11-9.

11 Farmer $\mathrm{AD}$, Coen SJ, Kano $\mathrm{M}$ et al. Psychophysiological responses to pain identify reproducible human clusters.
Pain 2013; doi:10.1016/j.pain.2013. 05.016. [Epub ahead of print].

12 Zigmond AS, Snaith RP. The hospital anxiety and depression scale. Acta Psychiatr Scand 1983; 67: 361-70.

13 Weinberger DA, Schwartz GE, Davidson RJ. Low-anxious, high-anxious, and repressive coping styles: psychometric patterns and behavioral and physiological responses to stress. $I$ Abnorm Psychol 1979; 88: 369-80.

14 Paine P, Kishor J, Worthen SF, Gregory LJ, Aziz Q. Exploring relationships for visceral and somatic pain with autonomic control and personality. Pain 2009; 144: 236-44.

15 Spielberger CD. Manual for the state/ trait anxiety inventory (form $Y$ ): (self evaluation questionnaire). Palo Alto: Consulting Psychologists Press, 1983.

16 Benarroch EE, Opfer-Gehrking TL, Low PA. Use of the photoplethysmographic technique to analyze the Valsalva maneuver in normal man. Muscle Nerve 1991; 14: 1165-72.

17 Eckert S, Horstkotte D. Comparison of Portapres non-invasive blood pressure measurement in the finger with intra-aortic pressure measurement during incremental bicycle exercise. Blood pressure monitoring 2002; 7: 179-83.

18 Cacioppo JT, Tassinary LG, Berntson GG. Handbook of Psychophysiology, 3rd edn. Cambridge: Cambridge University Press, 2007.

19 Julu PO. A linear scale for measuring vagal tone in man. I Auton Pharmacol 1992; 12: 109-15.

20 Julu PO, Cooper VL, Hansen S, Hainsworth R. Cardiovascular regulation in the period preceding vasovagal syncope in conscious humans. The Journal of physiology 2003; 549: 299-311.

21 Heart rate variability: standards of measurement, physiological interpretation and clinical use. Task Force of the European Society of Cardiology and the North American Society of Pacing and Electrophysiology. Circulation 1996; 93: 1043-65.
22 Cameron A, Henley D, Carrell R, Zhou A, Clarke A, Lightman S. Temperature-responsive release of cortisol from its binding globulin: a protein thermocouple. The Journal of clinical endocrinology and metabolism 2010; 95: 4689-95.

23 Kent P, Kongsted A. Identifying clinical course patterns in SMS data using cluster analysis. Chiropractic $\uplus$ manual therapies 2012; 20: 20.

24 Bacher J. SPSS twostep cluster: a first evaluation, 2004. Available at: http:// wwwstatisticalinnovationscom/products/twosteppdf. Accessed October 2012.

25 University of Leon. http://www.unileon.es/ficheros/servicios/informatica/ spss/english/IBM-SPSS_basico.pdf. Accessed October 2012.

26 Paine P, Worthen SF, Gregory LJ, Thompson DG, Aziz Q. Personality differences affect brainstem autonomic responses to visceral pain. Neurogastroenterol Motil 2009; 21: 1155-e98.

27 Dolnicar S. A review of unquestioned standards in using cluster analysis for data-driven market segmentation. Proceedings of the Australian and New Zealand Marketing Academy Conference 2002 CD Conference (ANZMAC 2002) 2002. University of Wollongong. Available at http:// ro.uow.edu.au/cgi/viewcontent.cgi? article $=1286 \&$ context $=$ commpapers, Accessed October 2012.

28 Formann AK, Kohlmann T. Latent class analysis in medical research. Stat Methods Med Res 1996; 5: 179 211.

29 Burke HM, Davis MC, Otte C, Mohr DC. Depression and cortisol responses to psychological stress: a meta-analysis. Psychoneuroendocrinology 2005; 30: 846-56.

30 Al Zoubi MB, Al Rawi M. An efficient approach for computing silhouette coefficients. Journal of computer science 2008; 4: 252-5.

31 Rao SS, Hayek B, Summers RW. Functional chest pain of esophageal 
origin: hyperalgesia or motor dysfunction. The American journal of gastroenterology 2001; 96: 2584-9.

32 Riedl A, Schmidtmann M, Stengel A et al. Somatic comorbidities of irritable bowel syndrome: a systematic analysis. I Psychosom Res 2008; 64: 573-82.

33 Willert RP, Woolf CJ, Hobson AR, Delaney C, Thompson DG, Aziz Q The development and maintenance of human visceral pain hypersensitivity is dependent on the N-methyl-Daspartate receptor. Gastroenterology 2004; 126: 683-92.

34 Norton GR, Norton PJ, Asmundson G), Thompson LA, Larsen DK. Neurotic butterflies in my stomach: the role of anxiety, anxiety sensitivity and depression in functional gastrointestinal disorders. I Psychosom Res 1999; 47: 233-40.

35 Clouse RE, Carney RM. The psychological profile of non-cardiac chest pain patients. Eur I Gastroenterol Hepatol 1995; 7: 1160-5.

36 Lightman SL. The neuroendocrinology of stress: a never ending story. $I$ Neuroendocrinol 2008; 20: 880-4.

37 Ritsner M, Gibel A, Maayan R et al. State and trait related predictors of serum cortisol to DHEA(S) molar ratios and hormone concentrations in schizophrenia patients. Eur Neuropsychopharmacol 2007; 17: 257-64.

38 Tak LM, Cleare AJ, Ormel J et al. Meta-analysis and meta-regression of hypothalamic-pituitary-adrenal axis activity in functional somatic disorders. Biol Psychol 2011; 87: 183-94.

39 Napadow V, Edwards RR, Cahalan $\mathrm{CM}$ et al. Evoked pain analgesia in chronic pelvic pain patients using respiratory-gated auricular vagal afferent nerve stimulation. Pain medicine 2012; 13: 777-89.

40 Zhang $\mathrm{X}, \mathrm{Cao} \mathrm{B}$, Yan $\mathrm{N}$ et al. Vagus nerve stimulation modulates visceral pain-related affective memory. Behav Brain Res 2013; 236: 8-15.

41 Botha C, Naqvi H, Chua YC, Knowles $\mathrm{CH}, \mathrm{Aziz} \mathrm{Q}$. Effect of autonomic modulation on human oesophageal pain hypersensitivity. Gut 2011; 60: A26.

42 Coppens CM, de Boer SF, Koolhaas JM. Coping styles and behavioural flexibility: towards underlying mechanisms. Philos Trans $R$ Soc Lond $B$ Biol Sci 2010; 365: 4021-8.

43 Shapiro MS, Siller S, Kacelnik A. Simultaneous and sequential choice as a function of reward delay and magnitude: normative, descriptive and process-based models tested in the European starling (Sturnus vulgaris). Journal of experimental psychology Animal behavior processes 2008; 34: 75-93.

44 Gwee KA, Graham JC, McKendrick MW et al. Psychometric scores and persistence of irritable bowel after infectious diarrhoea. Lancet 1996; 347: 150-3.

45 Chang L, Sundaresh S, Elliott J et al. Dysregulation of the hypothalamicpituitary-adrenal (HPA) axis in irritable bowel syndrome. Neurogastroenterol Motil 2009; 21: 149-59.

46 Mayer EA, Berman S, Suyenobu B et al. Differences in brain responses to visceral pain between patients with irritable bowel syndrome and ulcerative colitis. Pain 2005; 115: 398-409.

47 Kehlet H, Jensen TS, Woolf CJ. Persistent postsurgical pain: risk factors and prevention. Lancet 2006; 367: 1618-25.

48 Marshall JK, Thabane M, Garg AX et al. Incidence and epidemiology of irritable bowel syndrome after a large waterborne outbreak of bacterial dysentery. Gastroenterology 2006; 131: 445-50; quiz 660.

49 Zautra AJ, Fasman R, Davis MC, Craig AD. The effects of slow breathing on affective responses to pain stimuli: an experimental study. Pain 2010; 149: 12-8.

50 Pimentel M, Talley NJ, Quigley EM, Hani A, Sharara A, Mahachai V. Report from the multinational irritable bowel syndrome initiative 2012. Gastroenterology 2013; 144: e1-5.

51 Corsetti M, Caenepeel P, Fischler B, Janssens J, Tack J. Impact of coexisting irritable bowel syndrome on symptoms and pathophysiological mechanisms in functional dyspepsia. The American journal of gastroenterology 2004; 99: 1152-9. 